\title{
Differential Diagnosis of Neuroblastoma and Burkitt's Tumour
}

\author{
HILARY GRANT and R. J. V. PULVERTAFT \\ From the Departments of Paediatrics and of Pathology, University College Hospital, Ibadan, Western Nigeria
}

In Nigeria, as in most of tropical Africa, it is often impossible to determine the nature of a tumour involving the facial bones of a child, by clinical or radiological examination. The histology of a rapidly growing neuroblastoma has often been confused with other types of undifferentiated round cell malignant tumour, including retinoblastoma and Burkitt's tumour (African childhood lympho$\mathrm{ma}$ ). This case of neuroblastoma is described as an instance in which tissue culture provided an accurate diagnosis quickly, with minimal disturbance to the patient.

\section{Case Report}

S.A., a $1 \frac{1}{2}$-year-old Yoruba girl, was brought to University College Hospital Ibadan on April 15, 1965. Her mother had noticed a swelling of the left side of the face, which had been gradually enlarging for 3 months. Feeding had become difficult, and there had been some loss of weight.

She was a pale thin child weighing $16 \mathrm{lb}$. $(7 \cdot 25 \mathrm{~kg}$.) with a very large hard swelling of the left maxilla (Fig. 1a). The teeth had been loosened and displaced by tumour tissue, and the alveolar margin, which had an ulcerated bleeding surface, was protruding through the mouth. A firm mass with an indefinite border was felt in the left hypochondrium.

Received August 5, 1965.

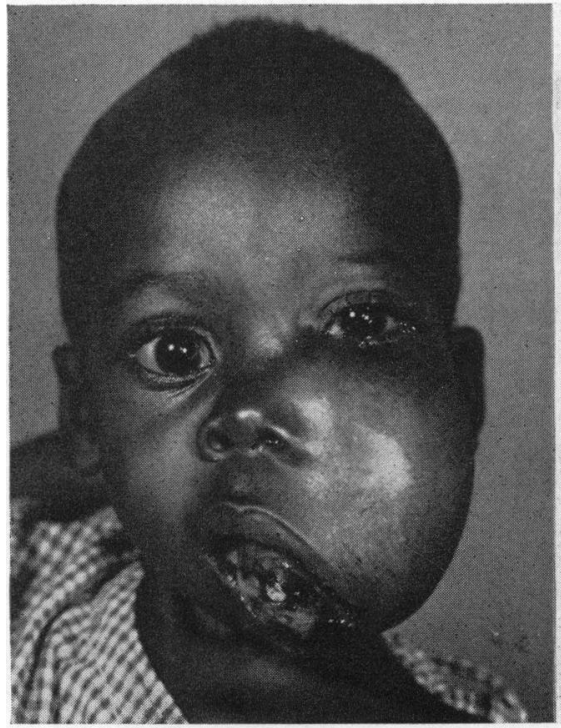

(a)

Fig. 1.-(a) Tumour of maxilla, neuroblastoma, secondary deposit.

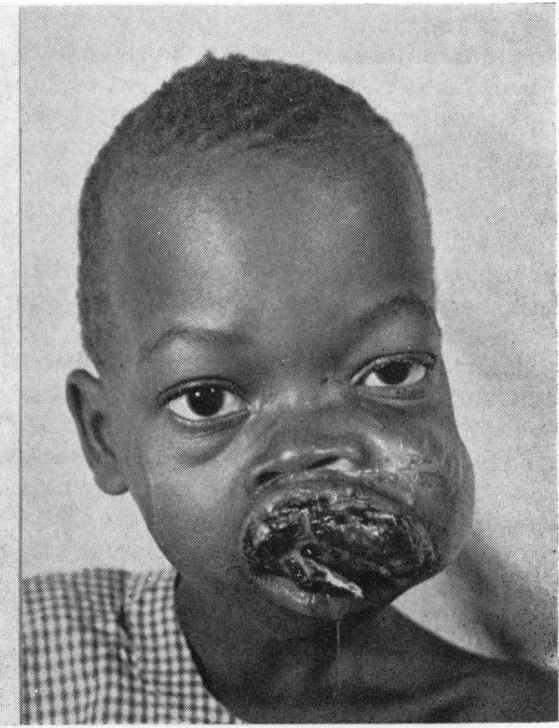

(b)

(b) Tumour of maxilla, Burkitt's tumour, primary growth. 
The tumour of the maxilla was indistinguishable on clinical examination from a typical Burkitt's tumour (Fig. 1b) which is by far the most common type of malignant tumour seen in children in Ibadan, accounting for $70 \%$ of all malignant disease in children under the age of 14 years (Edington and Maclean, 1964). The age of the patient caused doubt about this diagnosis, as no proved case of Burkitt's tumour has yet been seen in Ibadan in a child under 3 years, though three 2-year-old children were included by Burkitt (1958) in his original series from Kampala.

Investigations. Haemoglobin genotype AA. Blood group $B$ rhesus positive; haemoglobin 8.2 g. $/ 100 \mathrm{ml}$. (57\%); PCV 25\%; MCHC 33\%. WBC 8,600 per c.mm.; neutrophils $72 \%$, eosinophils $15 \%$, lymphocytes $13 \%$. Bone-marrow was megaloblastic with giant metamyelocytes; no tumour cells seen.

Radiographs of the facial bones showed considerable destruction of the left maxillary region with dental displacement and a large soft tissue tumour. This appearance was unhelpful in the differential diagnosis. A Burkitt's tumour involving the jaw may show typical radiological signs, including early loss of the lamina dura (Cockshott, 1964), but, as other neoplastic and inflammatory conditions can sometimes produce the same appearance, a diagnosis cannot be made on this evidence alone. An intravenous pyelogram showed the left kidney displaced downwards by a large suprarenal mass. This strongly suggested the diagnosis of neuroblastoma but did not exclude a Burkitt's tumour. The absence of tumour deposits visible on chest radiograph, and the presence of an osteolytic lesion in the left femoral neck would be consistent with the diagnosis of either neuroblastoma or Burkitt's tumour.

The diagnosis of neuroblastoma was established with certainty by tissue culture, as described below, within 24 hours of the patient being seen. A very small quantity of tumour tissue is required for this technique, and this was easily obtained from the maxillary tumour with an ordinary syringe and needle, without any special preparation of the patient.

Although the prognosis is known to be very poor when neuroblastoma is diagnosed after the age of 1 year, and when bone metastases are present (Gross, Farber, and Martin, 1959), it was decided to attempt treatment with cytotoxic drugs in addition to large doses of vitamin B12. Cyclophosphamide produced a rapid effect on the tumour cells as shown by the second tissue culture 40 hours after treatment was started, but the clinical response was disappointing. There was no decrease in the size of the tumour and the child's condition was deteriorating. Intravenous vincristine, started on May 12, 1965, seemed to produce a more favourable response. The child is still alive, with the abdominal and maxillary tumours decreasing in size, two months later.

\section{Tissue Culture}

According to Willis (1953), early in this century neuroblastomas were regarded as lymphosarcomas, though the history of early cases clearly discloses their true nature.

In Nigeria malignant tumours affecting the bones of the face include two, the neuroblastoma and retinoblastoma, which are well recognized in Europe, and one, the Burkitt tumour, which is common in children in Nigeria and certainly most unusual in Europe. All three tumours are composed of darklystaining cells with spherical nuclei, about $15 \mu$ to $20 \mu$ in diameter, and experience shows that sections of these tumours embedded in paraffin and stained with haematoxylin and eosin are frequently confused with each other. While both the retinoblastoma and the neuroblastoma may show rosettes, these are never found in Burkitt's tumour, and the rosette, when present, is a diagnostic feature of conclusive import. It is emphasized, however, that even in retinoblastomas and neuroblastomas, rosettes are only rarely found, and then only in slowly growing tumours.

Tissue culture as a diagnostic measure was first carried out by Murray and Stout (1947) on 8 cases including 2 adults in whom differential diagnosis was disputable. They were made in lying drops, and, since phase contrast was not then in routine use, the excellent illustrations are mainly of material stained by Bodian's method. Within 24 hours neurites were produced, and the value of tissue culture in distinguishing this tumour from lymphosarcoma and Ewing's tumour is stressed. By a coincidence, a paper by Lichtensten and Jaffe on Ewing's sarcoma appears in the same volume, and certainly supports the view that histology alone is not decisive in the classification of tumours consisting of spherical cells with a diameter of from $12 \mu$ to $20 \mu$. In this connexion it is noteworthy that no case of Ewing's sarcoma has been diagnosed in Nigeria (G. M. Edington, 1965, personal communication). Long-term culture, from 7 days to over a year, has been recorded by Goldstein, Burdman, and Journey (1964) with evidence in many cases of maturation and transformation into ganglion cells. The method used by them was explantation in fluid media or in thick plasma clot under perforated 'cellophane'; the fluid phase was $40 \%$ calf or human serum in 199 or modified Eagle's medium. The illustrations are very convincing, but the 'cellophane' layer somewhat interferes with critical optics.

Both in London and in Ibadan one of us (R.J.V.P.) has made a routine practice of examining material, suspected as being of neuroblastomatous origin, by some form of short-term tissue culture. Since, in most cases material comes from bone-marrow, it has usually been examined on agar in roller slides (Pulvertaft, 1965). Under these conditions neuro- 
blastoma cells are very characteristic, but difficult to photograph as they show very little phase contrast. They appear as chains or sheets of bright cells and mitose freely. Fibril formation is not convincing on agar; many cells, such as fibroblasts, may show single non-branching fibrils over $100 \mu$ long. Therefore, while on agar a diagnosis of a secondary malignant deposit can be made, it is less easy to be certain of its category.

Cellophane is not necessary for culture on glass, nor is a fibrin clot, as the cells adhere almost immediately and very firmly. Fibril formation, however, while freely developing on collagen, may not occur on glass (Pulvertaft, 1965).

In this case, remarkably free fibril development was found on glass.

Techniques. The material was received in a $10 \mathrm{ml}$. syringe on two occasions. About $2 \mathrm{ml}$. of heavily blood-stained semi-solid substance was available; under the microscope it was seen to consist almost entirely of coherent cells of uniform appearance and Burkitt's tumour was immediately excluded.

The cells were dispersed in a fluid medium by shaking. The medium consisted of $20 \%$ fresh adult human serum and $80 \%$ ascitic fluid from a case of nephrosis; nystatin and neomycin were added.

Results: first specimen. The tissue adhered almost immediately to the glass. In 18 hours long processes had already developed from the peripheral cells; several processes arose from each cell (Fig. 2a). During the following days the cell aggregates opened up, the constituent cells migrating peripherally. The intervening space became filled with a dense arborification of fine intercommunicating fibrils, approaching the limits of resolution (Fig. $2 b$ and $2 \mathrm{c}$ ). The fibrils terminated in an end-plate, with a webbing of cytoplasm (Fig. 2d). Later still fine short mitochondria and a few small granules travelled up the thickening fibrils.

Some of the fibrils were of great length, up to $300 \mu$. Where the cells were widely separated they showed clear evidence of reciprocal attraction, and when they met they appeared to fuse. Although critical microscopy could demonstrate no discontinuity in the single united fibril, granules were not seen to move from one cell to the other.

Under the microscope the advancing fibrils with their end-plates were often observed to cross another fibril at right angles. When this happened they never fused; the fibril went on extending until it met another end on; when this happened fusion appeared to be instantaneous. In the course of time both filamentous mitochondria and granules moved up the filament, but it was never possible to be certain that true continuity had been established. However, the appearances are contrasted with what is observed when two processes from adjacent fibroblasts make contact. In this case it seems clear that each cell remains as a functional and structural unit and organoids from one never enter the other. Only when a syncitium is formed, e.g. by giant cell formation in the presence of measles virus, has true cytoplasmic fusion been observed by one of us (R.J.V.P.).

When a cell entered mitosis, one daughter cell remained in fibrillar contact with others and the second extruded a fibril which soon made contact.

The cytoplasm contained very characteristic organoids (Fig. 3a). These were concentrated on one side only of the nucleus, and the group nearest the nucleus was poorly contrasted and appeared brown by phase. The peripheral groups were highly contrasted and appeared black.

The cultures were readily fixed and stained by Wigglesworth's osmic acid ethyl gallate technique, which closely imitates phase appearances. Here again the two groups of organoids were very obvious.

The medium was changed daily and mitosis increased in rate. Unfortunately on the sixth day all cultures became contaminated and died.

Second specimen. Two days after the initiation of cyclophosphamide therapy a second puncture of the jaw tumour was made. The material was identical in appearance with the first. It was cultured in the same medium and in the same manner as the first. In 18 hours, however, there was a most noticeable difference. About $80 \%$ of the cellular plaques were dead and did not adhere to the glass. The isolated cells extended fibrils as before, but the surviving cell aggregates never opened out and remained firmly as units; there were a few fibrils at the periphery. In this specimen many fibroblasts grew; none were seen in the first.

In 7 days all the cultures were dead; the fibrils were still visible floating loose in the fluid.

In the early preparations the neighbourhood of the cell aggregates showed a remarkable dense meshwork of very fine fibrils; they could only be resolved with a high performance objective (Fig. $3 b$ ).

A third puncture of the lesion was made two months later. Only a drop of material was procurable, and it consisted entirely of disintegrating polymorphs; no organisms were seen.

It will be seen from Fig. 1b that Burkitt's tumour may show a very close clinical resemblance to this case. Tissue culture (Fig. 3c) shows the typical 
(a)

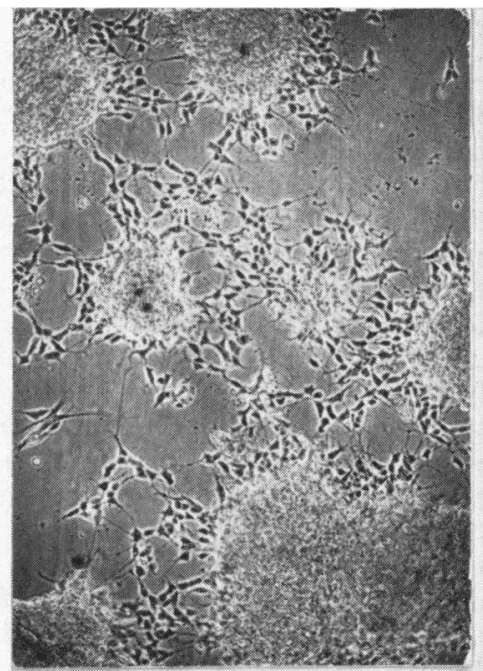

(c)

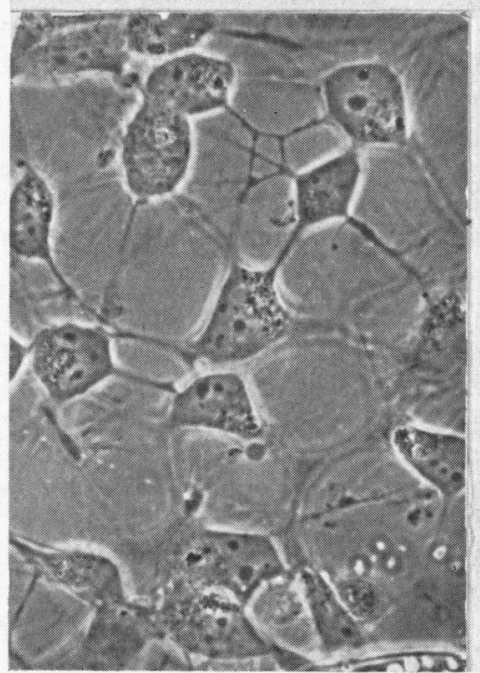

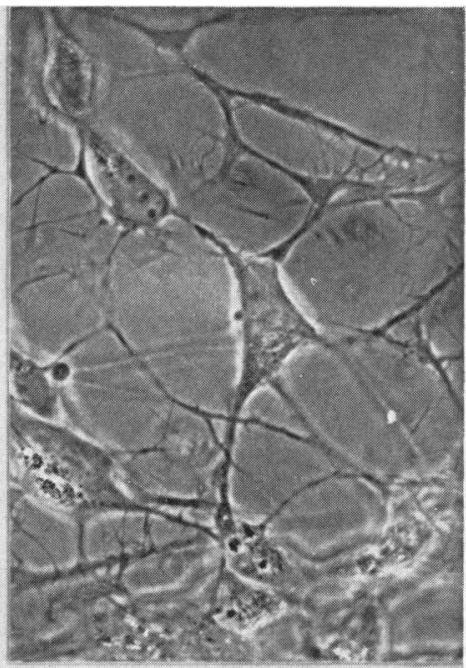

(b)

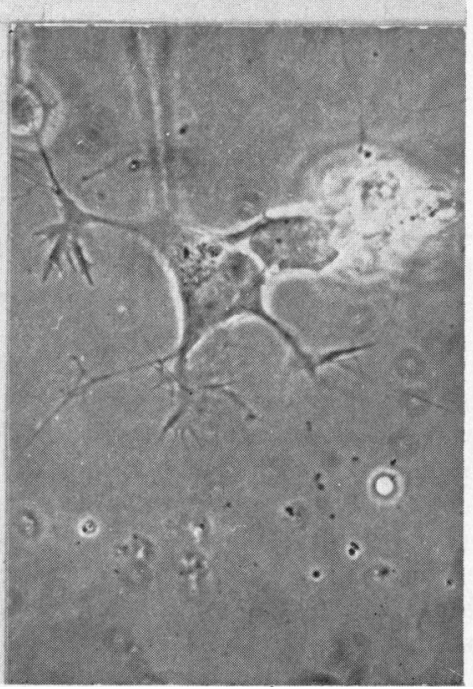

(d) spherical and granular cells, which are quite unmistakable. Our experience is that in most cases these cells can be obtained by needle puncture of the tumour, from ascitic fluid, or from the CSF, without submitting the child to an operation.

\section{Discussion}

The Burkitt tumour has perhaps attracted more attention than any neoplasm of childhood in recent years. Its peculiar geographical distribution and anatomical spread place it in a unique position. It is apparently of multifocal origin, and affects chiefly the bones, but also solid organs such as the ovary and kidney. When untreated, it is fatal in six months.

Recent developments in the therapy of malignant disease suggest that a combination of methods, including surgery, radiotherapy and chemotherapy may greatly modify the ultimate outcome, and that 'cure' may in certain cases be possible, even in conditions previously invariably and rapidly fatal. The position, therefore, can be compared with that which existed in the early days of antibiotics, when 


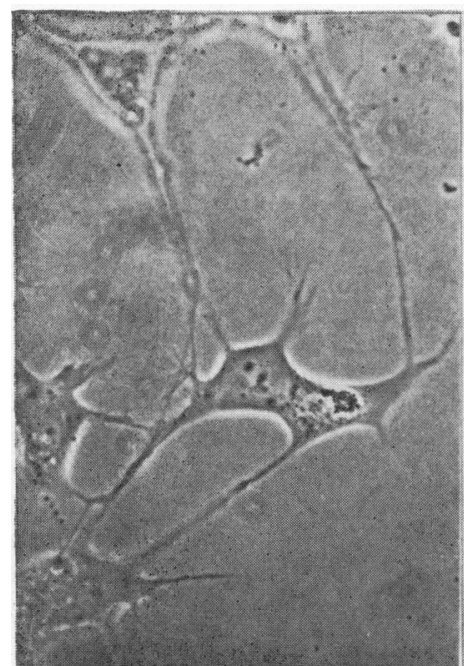

(a)

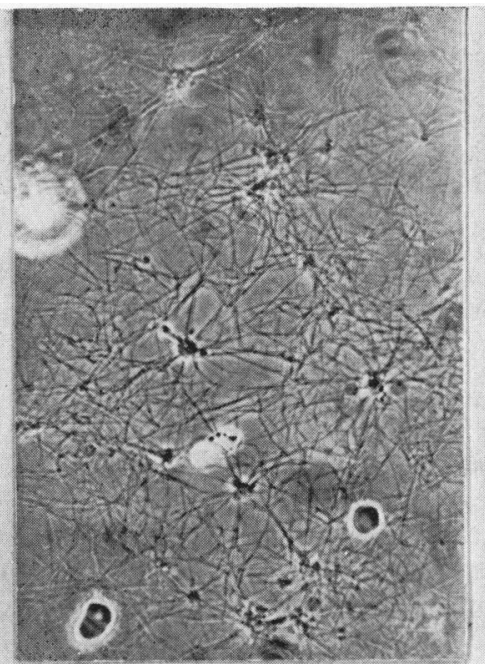

(b)

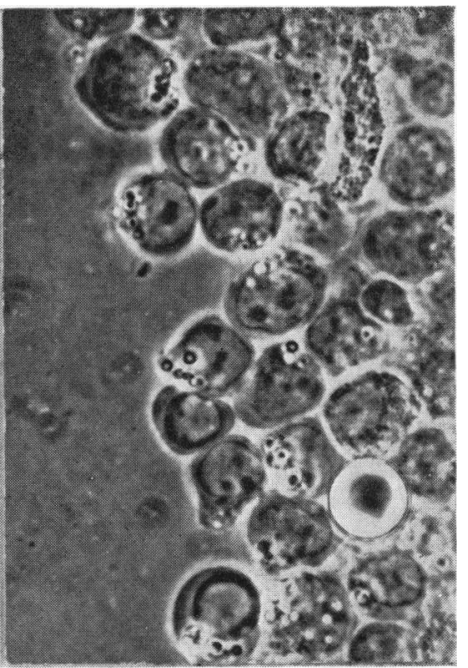

(c)

FIG. 3(a).-Neuroblastoma, juxtanuclear organoids. $(\times 1000).($ b) 48 hours after cyclophosphamide injection. ( $\times$ 1000.) (c) Burkitt's tumour; primary culture. ( $\times$ 1000.) All cultures photographed at $37^{\circ} \mathrm{C}$. by phase.

it became more than ever necessary to determine the infecting agent, and its sensitivity to a choice of reagents; or to the position in haematology 40 years ago, when the classification of the anaemias was an academic exercise.

For this reason the cytology of living cells and, in certain cases, simple tissue culture methods have an important place. They can provide not only a rapid, but also a definitive, diagnosis where conventional methods yield not only belated but also controversial diagnoses.

\section{Summary}

A case of neuroblastoma in an African child is described as an example of the value of tissue culture in the rapid differential diagnosis between it and Burkitt's tumour, with minimal disturbance to the patient.

The technique of tissue culture and the recognition of malignant neuroblastoma tissue are described.
Our thanks are due to the Department of Medical Illustration, University of Ibadan. The expenses of the investigation were defrayed by a full-time grant from the British Empire Cancer Campaign (R.J.V.P.). Professor G. M. Edington's help and advice were much valued. The technical work was carried out by Mrs. E. L. M. Pulvertaft.

\section{REFERENCES}

Burkitt, D. (1958). A sarcoma involving the jaws in African children. Brit. f. Surg., 46, 218.

Cockshott, W. P. (1964). Radiology of Burkitt's tumour in young Nigerians. In The Lymphoreticular Tumours in Africa, by F. C. Rowlet, pp. 150-162. S. Karger, Basle and New York. Edington, G. M., and Maclean, C. M. U. (1964). Incidence of the Burkitt tumour in Ibadan, Western Nigeria. Brit. med. $\mathcal{F}$., 1, 264.

Goldstein, M. N., Burdman, J. A., and Journey, L. J. (1964). Longterm tissue culture of neuroblastomas. II. Morphologic evidence for differentiation and maturation. $\mathcal{f}$. nat. Cancer Inst., 32, 165.

Gross, R. E., Farber, S., and Martin, L. W. (1959). Neuroblastoma sympatheticum. A study and report of 217 cases. Pediatrics, 23, 1179.

Murray, M. R., and Stout, A. P. (1947). Distinctive characteristics of the sympathicoblastoma cultivated in vitro. A method for prompt diagnosis. Amer. F. Path., 23, 429.

Pulvertaft, R. J. V. (1965). A study of malignant tumours in Nigeria by short-term tissue culture. $\mathcal{F}$. clin. Path., 18, 261.

Willis, R. A. (1953). Pathology of Tumours, 2nd ed., p. 843. Butterworth, London. 